\title{
A measure on the set of compact Friedmann-Lemaître-Robertson-Walker models
}

\author{
Boudewijn F Roukema $^{1}$ and Vincent Blanlœil ${ }^{2}$ \\ ${ }^{1}$ Torun Centre for Astronomy, Nicolaus Copernicus University, \\ ul. Gagarina 11, 87-100 Toruń, Poland \\ ${ }^{2}$ Département de Mathématiques, Université de Strasbourg, 7 \\ rue René Descartes, 67084 Strasbourg cedex, France
}

\begin{abstract}
Compact, flat Friedmann-Lemaître-Robertson-Walker (FLRW) models have recently regained interest as a good fit to the observed cosmic microwave background temperature fluctuations. However, it is generally thought that a globally, exactly-flat FLRW model is theoretically improbable. Here, in order to obtain a probability space on the set $F$ of compact, comoving, 3-spatial sections of FLRW models, a physically motivated hypothesis is proposed, using the density parameter $\Omega$ as a derived rather than fundamental parameter. We assume that the processes that select the 3-manifold also select a global mass-energy and a Hubble parameter. The requirement that the local and global values of $\Omega$ are equal implies a range in $\Omega$ that consists of a single real value for any 3 -manifold. Thus, the obvious measure over $F$ is the discrete measure. Hence, if the global mass-energy and Hubble parameter are a function of 3-manifold choice among compact FLRW models, then probability spaces parametrised by $\Omega$ do not, in general, give a zero probability of a flat model. Alternatively, parametrisation by a spatial size parameter, the injectivity radius $r_{\text {inj }}$, suggests the Lebesgue measure. In this case, the probability space over the injectivity radius implies that flat models occur almost surely (a.s.), in the sense of probability theory, and non-flat models a.s. do not occur.

PACS numbers: 98.80.Jk, 04.20.Gz, 02.40.-k
\end{abstract}




\section{Introduction}

Recent empirical analyses regarding the shape of the comoving spatial section of the Universe, assuming a perturbed Friedman-Lemaitre-Robertson-Walker (perturbedFLRW) model, have mostly focussed on the Wilkinson Microwave Anisotropy Probe (WMAP) all-sky maps of the cosmic microwave background. The results are presently inconclusive regarding both curvature and topology. The curvature has been found to be "nearly" zero, i.e., comoving space could be hyperbolic, flat or spherical. Writing the curvature as an effective density parameter $\Omega_{\mathrm{k}}:=1-\Omega$, where $\Omega$ is the total density parameter \pm none of the three possible curvatures $k \in\{0, \pm 1\}$ have been observationally excluded to high statistical significance. It is true that the precision in upper limits to $\left|\Omega_{\mathrm{k}}\right|$ has improved by about one a half orders of magnitude in the last decade and a half, and that the accuracy in $\left|\Omega_{\mathrm{k}}\right|$ upper limits has similarly improved, but these limits do not exclude any of $k \in\{0, \pm 1\}$.

Results regarding topology, i.e. the fundamental group $\Gamma$ of holonomy transformations of the comoving spatial section, are also inconclusive. Several groups have found that the Poincaré dodecahedral space $S^{3} / I^{*}$, with covering space $\widetilde{M}=S^{3}$ and fundamental group $\Gamma=I^{*}$, is preferred over simply connected infinite flat space (Luminet et al., 2003; Aurich et al., 2005a.b; Gundermann, 2005; Caillerie et al., 2007; Roukema, Buliński, Szaniewska and Gaudin, 2008; Roukema, Buliński and Gaudin, 2008) in order to fit the WMAP data, while others exclude detectable cosmic topology (Cornish et al., 2004; Kev et al., 2007), or prefer simply connected infinite flat space (Niarchou and Jaffe, 2007; Lew and Roukema, 2008).

While a well-established theoretical basis for cosmic curvature exists (the Einstein field equations match the spatial curvature of the Universe to its matter-energy density), there are at present only hints as to what might constitute a theory of cosmic topology. Various quantum gravity aspects of cosmic topology include the decay from pure quantum to mixed states (e.g., Hawking, 1984), smooth topology evolution (e.g., Dowker and Surya, 1998), and work that could contribute to a physical motivation for deciding which 3-manifold should be favoured by a theory of quantum cosmology (Seriu, 1996; Anderson et al., 2004). A recent heuristic result is that of the dynamical effect of cosmic topology in the presence of density perturbations. A residual weak-limit gravitational effect in the presence of a density perturbation selects well-proportioned spaces (Weeks et al., 2004) in general, and the Poincaré dodecahedral space $S^{3} / I^{*}$ in particular, to be special in the sense of being "better balanced" (Roukema et al., 2007; Roukema and Różański, 2009). The agreement between the residual gravity effect and many of the empirical analyses in choosing the same space, the Poincaré space, suggests that this effect might have been important in the early universe in selecting the comoving

$\ddagger$ The total density parameter is defined $\Omega:=\rho_{\text {tot }} / \rho_{\text {crit }}$, where $\rho_{\text {tot }}$ and $\rho_{\text {crit }}$ are the total density and the critical density, respectively. The latter is $\rho_{\text {crit }}:=3 H^{2} /(8 \pi G)$ where $H$ is the Hubble parameter and $G$ the gravitational constant. 
3-manifold in which we live.

On the other hand, Aurich et al. (Aurich et al., 2007; Aurich, 2008; Aurich et al., 2008, 2010) have recently carried out several studies showing that a flat compact model (specifically, $T^{3}$ ) generally provides a better fit to the WMAP data than infinite flat models. This work provides one of the few ways of potentially showing that the Universe is flat in the sense of being a flat 3-manifold (apart from perturbations), if that is indeed the true shape of the Universe, rather than a Poincaré space. Approaches that ignore topology sometimes argue in favour of flatness while simultaneously stating (correctly) that they cannot determine the curvature. For example, Vardanyan et al. (2009) calculate the odds "in favour of a flat Universe", but also state that "the [curvature] of the Universe is not knowable" if $\log _{10}\left|\Omega_{\mathrm{k}}\right|<-4$, and make no claim that $\log _{10}\left|\Omega_{\mathrm{k}}\right| \geq-4$. That is, they assert that either the curvature has not yet been determined, or it is unknowable. In contrast, use of the global properties of the comoving spatial section potentially offers a way to determine the curvature, even if the curvature is zero, as suggested by Aurich et al.'s work, and even in the (realistic) presence of perturbations.

Although flat, compact space models have been frequently modelled, they have generally been disliked for fine-tuning reasons, especially in relation to inflationary motivations and scenarios. It is frequently stated that the probability of the spatial section of the Universe, according to the exact-FLRW model, being exactly flat is zero. For example, in a discussion about common misconceptions regarding "standard universe models" and inflation, Ellis (2006) states that being exactly flat "requires infinite fine tuning of initial conditions; if say the two millionth digit in the value of $\Omega_{\mathrm{k}}$ is non-zero at any time, then the universe is not spatially flat" and that "although the scale-free $k=0$ exponential case is the model underlying the way many people approach the problem, it is highly exceptional - it is of zero measure within the space of all inflationary FL models" (Sect. 2.8.1, 2.7.1, resp., Ellis, 2006). That is, if the measure $\mu$ on the space $F$ of all exact-FLRW models is a probability measure, i.e. if $\mu(F)=1$, then the probability of exact flatness is zero: $P(k=0)=\mu(k=0)=0$. Since inflationary scenarios only favour near flatness, not exact flatness, the subclass of inflationary scenarios $I \subset F$ can validly have $\mu(I)>0$, i.e. a positive probability, according to this implicit definition of a measure space $(F, \Sigma, \mu)$.

However, is being exactly flat necessarily a physical possibility that has zero measure? Ellis and van Elst (1999) argue that finding a natural, plausible, measure in the "full space of cosmological space-times" is an open problem. Previous suggestions of measures (e.g., Gibbons et al., 1987; Coule, 1995; Evrard and Coles, 1995; Kirchner and Ellis, 2003; Gibbons and Turok, 2008) have typically only considered the curvature of the spatial section and not the fact that it is a 3-manifold that has both a curvature and a topology. In other words, the set $F$ of exact-FLRW models is considered to be equivalent to $F_{\Gamma=0}:=\{\Omega \in \mathbb{R}: \Omega>0\}$, i.e. it is parametrised only by the density parameter $\Omega$. The measure is implicitly assumed to be a simple function of the Lebesgue measure on $F_{\Gamma=0}$ that gives $\mu(\Omega=1)=0$ and a normalised measure $P\left(F_{\Gamma=0}\right)=1$, so 
that $P(\Omega=1)=0$.

The fact that an FLRW model has both a curvature and a topology is not a new insight. Both curvature and topology have been mentioned quite clearly by several of the founders of relativistic cosmology (de Sitter, 1917; Friedmann, 1923, 1924; Lemaître, 1931; Robertson, 1935). The curvature and topology of FLRW models have usually been thought to be independent of one another, except that the set of possible 3-manifolds is divided between hyperbolic, flat, and spherical 3-manifolds. Some work that does consider topology includes that of Seriu (1996) and Anderson et al. (2004). Seriu (1996) defined a "spectral distance" function over pairs of universes with (in general) different spatial sections, but did not try extending this to a measure. Anderson et al. (2004) applied a Hartle-Hawking "no boundary" path integral approach to the spatial section that constitutes a boundary to a global space-time. Alternatively, a Bayesian approach to measure spaces and the flatness problem, using Jaynes' principle, finds that the flatness problem is not a problem (Evrard and Coles, 1995; Kirchner and Ellis, 2003).

Here, a more elementary approach to obtaining a measure on the set of exactFLRW models, considering both the curvature and topology of the models, is presented. In Sect. 2.1, a physically motivated hypothesis is proposed. This hypothesis is used to determine the set of compact, 3-spatial sections of exact-FLRW models where the density parameter $\Omega$ is a derived rather than fundamental physical parameter. Only compact models are considered, with the motivation that this is physically more reasonable than infinite models $\$$ The resulting set and the natural measure on it are presented in Sect. 2.4. An alternative definition of the set, using the injectivity radius $r_{\text {inj }}$ (half the length of the shortest closed comoving spatial geodesic), and the natural measure on it are discussed in Sect. 3. Conclusions are given in Sect. 4.

For early references to cosmic topology see Schwarzschild (1900) Friedmann (1923, 1924); Lemaître (1931) and Robertson (1935). For a short, modern introduction see Roukema (2000). For reviews, see Lachièze-Rey and Luminet (1995); Luminet (1998); Starkman (1998); Luminet and Roukema (1999); Blanloeil and Roukema (2000); Rebouças and Gomero (2004).

\section{Parametrisation by $\Omega$}

\subsection{Hypothesis}

The implicit assumption that the space of exact-FLRW models consists of $F_{\Gamma=0}:=\{\Omega \in$ $\mathbb{R}: \Omega>0\}$ is a statement that $\Omega$ is a free parameter, unconstrained by prior physics. One way of introducing some physical motivation for a theory of a set of possible universes is to extrapolate from known physics.

Local geometry is a fundamental and experimentally well-established part of modern physics, via the Einstein field equations. It would be physically reasonable

$\S$ For hyperbolic models, replacing the condition "compact" by "finite volume", i.e. allowing finite volume models containing infinite spatial geodesics, should not modify the results in this paper.

\| English translation: Stewart et al. (1998). 
that as a result of quantum gravity processes or during (mostly) smooth early-universe topology evolution as explored by Dowker and Surya (1998), some global physical properties of the Universe in the FLRW approximation (not necessarily the density $\Omega$ ) could be determined by a global geometrical property. The most obvious global geometrical property is geometria situs (Euler, 1736), known today as topology. For exact-FLRW models, the topological characteristic that is usually discussed is equivalent to the choice of constant curvature 3-manifold.

What property or properties could most reasonably be hypothesised to be determined by the choice of topology? Although density (in an exact-FLRW model) can be evaluated globally, a more fundamental property in a classical, pre-relativistic sense, is mass. In pre-relativistic physics, conservation of mass in any closed system is a fundamental principle. In FLRW cosmological models, let us define the global (by volume), total (by component) non-relativistic plus relativistic mass-energy in the 3 -spatial section as $m:=\rho_{\text {tot }} V^{\prime}$, where $\rho_{\text {tot }}$ is the total (by component) mass-energy density, and $V^{\prime}$ is the volume of the spatial section in physical units, if $k=0$, and as $m:=\rho_{\text {tot }} V R_{\mathrm{C}}^{3}$, where $R_{\mathrm{C}}$ is the curvature radius in physical units and $V$ is the volume of the spatial section in units of $R_{\mathrm{C}}^{3}$, if $k \neq 0$. Since, in general, $m$ is not conserved with time, due to the change in frequency of (in particular) photons with respect to locally comoving observers, this should be evaluated at a given epoch.

Another parameter that could reasonably depend on the choice of 3-manifold is the Hubble parameter $H$. Thus, along with the above definition of $m$, the following hypothesis is proposed.

Hypothesis 1 The topological evolution processes in the early universe that determine the comoving spatial 3-manifold $M$ of an FLRW universe lead to, at a given posttopology-evolution epoch $t_{\mathrm{t}}$, a global, tota matter-energy $m(M):=\left.m(M)\right|_{t_{\mathrm{t}}}$, and a rate of expansion, i.e. the Hubble parameter $H(M):=\left.H(M)\right|_{t_{\mathrm{t}}}=\left.(\dot{a} / a)(M)\right|_{t_{\mathrm{t}}}$. That is, $m$ and $H$ at the epoch $t_{\mathrm{t}}$ are functions from the space of exact-FLRW models $F$ to $\mathbb{R}$, written $m(M), H(M)$ to denote this dependence. The total mass-energy $m(M)$ is determined in the comoving frame. The manifold $M$ is assumed to be compact and orientable, and to be fixed for all epochs at $t>t_{\mathrm{t}}$.

The epoch $t_{\mathrm{t}}$ is thought of here to be pre-inflationary if an inflationary scenario is invoked, but neither requires nor rejects an inflationary epoch at $t>t_{\mathrm{t}}$. To investigate possible measure spaces using Hypothesis 1, spatially curved and flat models need to be considered separately.

\subsection{Curved models}

For curved models, first let us consider the density parameter $\Omega$ as a local parameter, i.e. as a limit towards a space-time point. In order to have a real, positive curvature

I The term "global" refers to the whole of comoving space, while "total" refers to all of the matterenergy density components, such as baryons, non-baryonic dark matter, neutrinos, radiation, and dark energy. 
radius $R_{\mathrm{C}}$ for both negative $(k=-1)$ and positive $(k=+1)$ curvature, let us define $R_{\mathrm{C}} \in \mathbb{R}$ such that $R_{\mathrm{C}}>0$ and the Friedmann equation is

$$
\Omega=1 \pm\left(\frac{c}{H(M) R_{\mathrm{C}}}\right)^{2}, k= \pm 1
$$

In a compact, exact-FLRW model, $\Omega$ also has a global meaning. Let us write the volume of a compact 3-manifold $M$ of constant, non-zero curvature and (real) curvature radius $R_{\mathrm{C}}>0$ as $V R_{\mathrm{C}}^{3}$, i.e. $V$ is dimensionless. For example, the lens space $L(p, q)$

for $p, q \in \mathbb{Z}, 0<q \leq p / 2$, with $p, q$ relatively prime (e.g., Gausmann et al., 2001), has volume $2 \pi^{2} R_{\mathrm{C}}^{3} / p$, so that $V[L(p, q)]=2 \pi^{2} / p$. In the hyperbolic case, the uniqueness of $V$ for a given fundamental group $\Gamma$ was less obvious than for the spherical case, but follows from Mostow (1968)'s rigidity theorem + The definitions of the critical density and the density parameter give

$$
\Omega:=\frac{\rho}{\rho_{\text {crit }}}=\frac{m(M)}{V R_{\mathrm{C}}^{3}} \frac{8 \pi G}{3[H(M)]^{2}} .
$$

What values of $\Omega$ simultaneously satisfy (10) and (2)? Clearly, the local and global values of the density parameter must be equal in an exact-FLRW model. Hence,

$$
1 \pm\left(\frac{c}{H(M) R_{\mathrm{C}}}\right)^{2}=\frac{m(M)}{V R_{\mathrm{C}}^{3}} \frac{8 \pi G}{3[H(M)]^{2}},
$$

where \pm correspond to curvatures $k= \pm 1$, respectively, as above. The range of $R_{\mathrm{C}}$ values that satisfy this equation, and in turn, the values of $\Omega$ that satisfy both (1) and (2) for a given pair $[m(M), H(M)]$, are presented in Sect. 2.4.

\subsection{Zero spatial curvature}

The equivalent of (1) for a flat, compact, exact-FLRW model is

$$
\Omega=1 .
$$

Hence, the equivalent of (2) is not needed in order to determine the valid range of $\Omega$ for flat models.

\subsection{The set $F_{m, H}^{\Omega}$ of FLRW models parametrised by $\Omega$}

The set $F$ of compact, exact-FLRW models is considered here to be the set of compact, comoving spatial sections that have an FLRW metric. A superscript $\Omega$ is used to indicate the parameter over which a measure space is to be defined. The subscripts $m, H$ indicate that Hypothesis 1 is assumed.

Equation (3) is a cubic equation in $R_{\mathrm{C}}$. The discriminant is

$$
\Delta=-324 V^{2} H(M)^{2}\left( \pm c^{6} V^{2}+48 \pi^{2} G^{2} m(M)^{2} H(M)^{2}\right) .
$$

For positive curvature, $\Delta<0$, so there is only one real root for $R_{\mathrm{C}}$. For negative curvature, there can be up to three distinct, real roots, but from (11) and (2), it is clear

+ Extended to the non-compact, finite volume case by Prasad. 


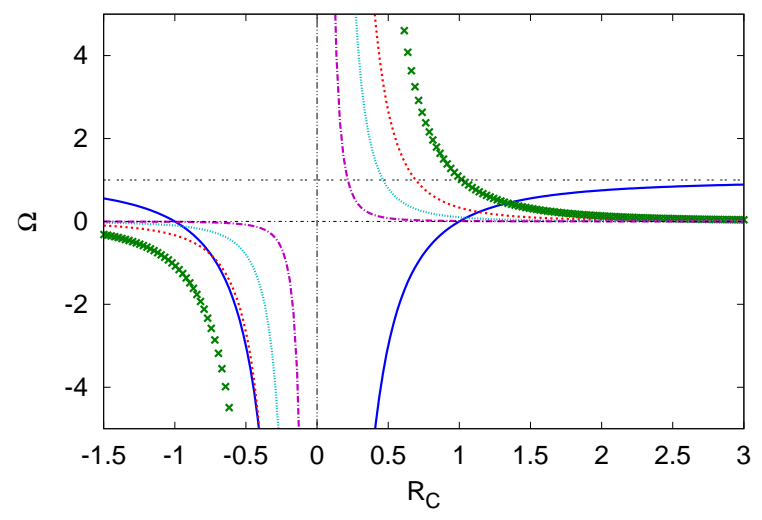

Figure 1. Example of local and global dependence of the (total) density parameter $\Omega$ on the curvature parameter $R_{\mathrm{C}}$ in hyperbolic, compact FLRW models, given Hypothesis 1, in the special case where $m(M)$ and $H(M)$ are constants independent of $M$. The local definition of the density parameter (1) gives the pair of curves that are symmetric around $R_{\mathrm{C}}=0$ and decrease without bound as $R_{\mathrm{C}} \rightarrow 0$ (solid curves). The global definition of the density parameter (2) is shown for dimensionless volume $V=0.943$ (" $\times$ " symbols; this is for the smallest-volume orientable hyperbolic 3manifold, the Weeks-Matveev-Fomenko manifold) and for $V=3.0,10.0$, and 100.0. The unphysical range $R_{\mathrm{C}} \leq 0$ is shown for algebraic completeness, in order to show the cases where one or more unphysical solutions with $R_{\mathrm{C}}<0$ occur. The units are chosen so that $c=H(M)=8 \pi G m(M) / 3=1$, i.e. $R_{\mathrm{C}}$ is in units of $c / H(M)$.

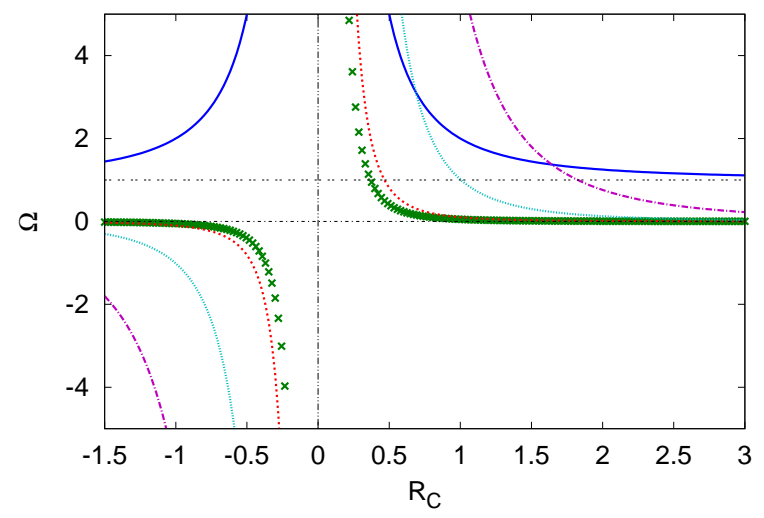

Figure 2. As for Figure 1, for spherical models. The local definition of the density parameter (11) gives the pair of curves symmetric around $R_{\mathrm{C}}=0$ that increase without bound as $R_{\mathrm{C}} \rightarrow 0$ (solid curves). The global definition of the density parameter (2) is shown for $V=2 \pi^{2}$ ("×" symbols; this is for the largest spherical 3-manifold $S^{3}$ ) and $V=\pi^{2}, \pi^{2} / 10$, and $\pi^{2} / 60$. The unphysical range $R_{\mathrm{C}} \leq 0$ yields no solutions. 
that only one of these is positive. This is illustrated in Figures 1 and 2. The multiple root or the two additional roots that can occur in the hyperbolic case (Figure 1) occur for $R_{\mathrm{C}}<0$, which is unphysical given the definition of $R_{\mathrm{C}}$ used here**

For illustrative purposes, we can consider the case where $m(M)$ and $H(M)$ are equal for all 3-manifolds. Figure 1 shows that in this case, for hyperbolic 3-manifolds, there is a maximum possible curvature radius $R_{\mathrm{C}}$, i.e. a maximum possible density parameter $\Omega$, at $t_{\mathrm{t}}$. These maxima are attained for the smallest-volume hyperbolic 3-manifold. If we consider only orientable, hyperbolic 3-manifolds, then the Weeks-Matveev-Fomenko

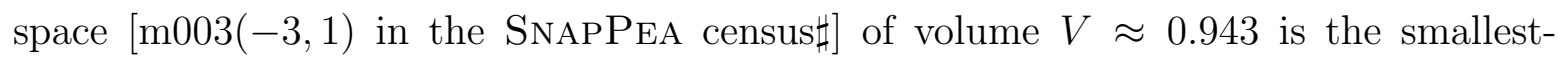
volume orientable hyperbolic 3-manifold (Weeks, 1985; Matveev and Fomenko, 1988; Gabai et al., 2009), shown here by " $\times$ " symbols. Larger volume hyperbolic 3-manifolds give the continuous curves in the figure [shown for $V \approx 3.0$, e.g. $\operatorname{m117}(-5,2)$ in the SNAPPEA census, and for $V=10.0,100.0]$, which successively give lower $R_{\mathrm{C}}$ and $\Omega$. In Figures 1 and 2, not only are $m(M)$ and $H(M)$ fixed, but the displayed ranges of numerical values of $R_{\mathrm{C}}$ and $\Omega$ have been chosen for convenience only, i.e. with the units $c=H(M)=8 \pi G m(M) / 3=1$. Elsewhere, these parameters are considered to retain their physical units.

Similarly, Figure 2 shows that for the spherical case, fixed values of $m(M)$ and $H(M)$ independent of $M$ imply a minimum possible curvature radius $R_{\mathrm{C}}$ as the choice of 3-manifold (i.e. $\Gamma$ ) is varied, which corresponds to a maximum possible density parameter $\Omega$. These are attained for the largest-volume spherical 3-manifold, i.e. $S^{3}$ itself, of volume $V=2 \pi^{2}$. While both signs of the curvature give upper limits to $\Omega$ for fixed $m(M)$ and $H(M)$ as $\Gamma$ varies, the hyperbolic case prevents $\Omega$ from approaching the flat case, while the spherical case allows $\Omega$ to approach arbitrarily close to flatness as the volume decreases. The volume $V=\pi^{2} / 60$ is that of the Poincaré dodecahedral space.

More generally, i.e. without fixing $m(M)$ and $H(M)$, the unique physical solution for $R_{\mathrm{C}}$ in both cases is

$$
R_{\mathrm{C} \pm}(M)=\frac{\left(\sqrt{ \pm c^{6} V^{2}+48 \pi^{2} m^{2} G^{2} H^{2}}+4 \sqrt{3} \pi m G H\right)^{\frac{2}{3}} \mp c^{2} V^{\frac{2}{3}}}{\sqrt{3} H V^{\frac{1}{3}}\left(\sqrt{ \pm c^{6} V^{2}+48 \pi^{2} m^{2} G^{2} H^{2}}+4 \sqrt{3} \pi m G H\right)^{\frac{1}{3}}}
$$

where the dependence of $R_{\mathrm{C}}$ on the manifold $M$ occurs through the dependences $V(M), m(M)$, and $H(M)$.

The density parameter for a curved FLRW model can be written using either (1) or (2). The former gives

$$
\Omega_{ \pm}(M)=1 \pm\left[\frac{c}{H(M) R_{\mathrm{C}_{ \pm}}(M)}\right]^{2}
$$

where $M=\mathbb{H}^{3} / \Gamma$ or $M=S^{3} / \Gamma$, and $R_{\mathrm{C}_{ \pm}}$is given in (6). As noted above (4), the

* An alternative definition could use imaginary values of $R_{\mathrm{C}}$ for the hyperbolic case.

\# The file/usr/share/snappea/ClosedCensusData/ClosedCensusInvariants.txt of version 3.0d320 of SNAPPEA is referred to in this paper. 
solution in the flat case is

$$
\Omega_{k=0}(M)=1 \text {. }
$$

Together, these give the following result. For any given manifold $M$, Hypothesis 1 implies a single value of $\Omega$ rather than a continuous interval of possible values of $\Omega$. In other words, there is no freedom in choosing $\Omega$ once $M$ has been selected. Since the set of constant curvature manifolds $\{M\}$ is countable (e.g., Lachièze-Rey and Luminet, 1995), we can write $F_{m, H}^{\Omega}$ as the countable (infinite) set

$$
F_{m, H}^{\Omega}=\{\Omega(M): M \in F\},
$$

where $\Omega(M)$ is given by (7) and (8) .

\subsection{Measure spaces and probability spaces}

Let us choose the obvious $\sigma$-algebra, $2^{F_{m, H}^{\Omega}}$, i.e. the set of all subsets of $F_{m, H}^{\Omega}$, and any discrete measure $\mu$ on $\mathbb{R}$ satisfying

$$
\begin{aligned}
& \mu(\Omega)>0, \Omega \in F_{m, H}^{\Omega} \\
& \mu(\Omega)=0, \Omega \notin F_{m, H}^{\Omega} .
\end{aligned}
$$

Then $\left(F_{m, H}^{\Omega}, 2^{F_{m, H}^{\Omega}}, \mu\right)$ is a measure space over the density parameter $\Omega$ of exact-FLRW models of the Universe. It is clear that there is no physical requirement that $\mu(\Omega=1)$ be zero, i.e. there is no requirement that the measure of the case of a flat FLRW model be zero.

For a probability space $\left(F_{m, H}^{\Omega}, 2^{F_{m, H}^{\Omega}}, \widehat{\mu}\right)$ to be made starting from the measure $\mu$, it would be required that $\widehat{\mu}\left(F_{m, H}^{\Omega}\right)=1$. Let the manifolds be enumerated $\left\{M_{i}\right\}_{i=1}^{\infty}$ and $\Omega(M)$ as given by (7) and (8) be enumerated $\Omega_{j}$, where a non-bijective function $j(i)$ is provided so that $j_{1} \neq j_{2} \Rightarrow \Omega_{j_{1}} \neq \Omega_{j_{2}}$ i.e. manifolds of equal $\Omega$ are included as a single case with respect to the measure. (Otherwise, $\widehat{\mu}\left(\Omega_{j}\right)$ would be multiply defined for some values of $\Omega_{j}$, e.g. $\Omega_{j}=1$.) Any convergent series $\left\{x_{j}\right\}_{j=1}^{\infty}$ with $\Sigma_{j} x_{j}=1$ now satisfies

$$
\begin{array}{ll}
\widehat{\mu}\left[\Omega_{j}\left(M_{i}\right)\right]=x_{j}, & M_{i} \in F \\
\widehat{\mu}(\Omega)=0, & \Omega \notin F_{m, H}^{\Omega},
\end{array}
$$

giving a probability space $\left(F_{m, H}^{\Omega}, 2^{\left.F_{m, H}^{\Omega}, \widehat{\mu}\right)}\right.$.

For example, this would be satisfied by

$$
\begin{array}{lr}
j=1, x_{j}=1 / \mathrm{e}, & 1 \leq i \leq 6 \\
j \geq 2, x_{j}=1 /[(j-1) ! \mathrm{e}] & , i \geq 7 \\
M_{i}=\mathrm{E}_{i}, & 1 \leq i \leq 6,
\end{array}
$$

where $\mathrm{E}_{i}$ are the six compact, flat, orientable 3-manifolds as labelled in Table I of Riazuelo et al. (2004), and the non-flat, compact, orientable 3-manifolds are enumerated by $i \geq 7, j(i) \geq 2$. This gives

$$
\widehat{\mu}(\Omega=1)=\widehat{\mu}\left(\Omega_{1}\right)=x_{1}=1 / \mathrm{e} \approx 0.37>0
$$


That is, given this example of $\left\{x_{j}\right\}$, the probability of a flat space would be about $37 \%$, i.e. strictly greater than zero. No physical motivation is suggested for this particular choice of $\left\{x_{j}\right\}$ and enumeration $\left\{M_{i}\right\}$ of the 3-manifolds. This choice of $\left\{x_{i}\right\}$ and corresponding (partial) enumeration $\left\{M_{i}\right\}$ are presented only as a numerical example of how $P(\Omega=1)>0$ is possible, with $P(\Omega=1) \equiv \widehat{\mu}(\Omega=1)=1 /$ e. It would remain possible to choose $\left\{x_{j}\right\}$ in a way that gives $P(\Omega=1)=0$, but it would be not be necessary to do so. Additional physical assumptions would be required to determine what the normalised discrete measure $\widehat{\mu}$ should be. Alternatively, $\Omega$ might not be a physically useful choice for defining a measure space. The construction in Sect. 3 suggests one alternative.

\subsection{How can $\Omega=1$ not be a case of measure zero?}

Fine-tuning arguments have played a role in cosmology for (at least) several decades. It may seem strongly counterintuitive that exactly $\Omega=1$ models do not necessarily constitute a case of measure zero, since the number 1 is a single real number on the continuous real number line. How does the topology of spatial sections invalidate this argument? The reason is that while topology in some sense might appear to give "additional" parameter freedom to the FLRW models, curvature can be thought of as a type of rigidity that together with topology reduces a continuous interval in the possible range of $\Omega$ to a set of discrete points.

Thus, the hypothesis presented here leads to a physical link between curvature and topology. This link is represented algebraically in (3). This equation represents a physical relation between curvature and topology implied by Hypothesis 1. For a given compact manifold $M$, the relativistic mass-energy $m(M)$ must be distributed uniformly throughout the space, expanding at the given rate $H(M)=(\dot{a} / a)(M)$, in a way that simultaneously gives the curvature implied locally at each point in comoving space by the Friedmann equation and fills the volume implied by the global shape of the manifold. The local and global roles of the density $\Omega$ do not have the freedom to be unrelated to one another if $m$ and $H$ are determined by the choice of 3-manifold.

The full mathematics that underlies this physical argument has only become known in the second half of the twentieth century, and some details even more recently. The complete classification of spherical 3-manifolds and Mostow (1968)'s rigidity theorem for compact, hyperbolic 3-manifolds play a role by implying a unique volume $V$ for any given curved, compact 3-manifold. A proof that the Weeks-Matveev-Fomenko manifold is the smallest-volume orientable hyperbolic 3-manifold was only published in 2009 (Gabai et al., 2009).

It is this physical relation between $\Omega$ being both local and global, which is a result of the rigidity of (constant curvature) curved 3-manifolds, that reduces the apparent continuum of values of $\Omega$ in the curved case to a discrete set. The set of values of $\Omega$ in the flat case is already discrete, since it contains just one member, $\Omega=1$. Hence, the full set of allowed values of $\Omega$ is discrete, and the natural measure is a discrete measure 
$\mu$, as indicated in (10), rather than the Lebesgue measure. This is why $\Omega=1$ need not be a case of measure zero.

\section{Parametrisation by a one-dimensional size parameter}

Moreover, while Hypothesis 1 reduces the freedom of $\Omega$ in curved FLRW models, it implies more parameter freedom in flat FLRW models for other parameters.

In the 3-torus model $T^{3}$, there are (at least) three parameters required to define the fundamental domain. Following Lehouca et al. (1996), we can write the size of the domain as $L_{\mathrm{a}} L_{\mathrm{e}} L_{\mathrm{u}}$. For orthogonal side-lengths of the fundamental parallelepiped of a $T^{3}$ model, the definition of the critical density gives

$$
L_{\mathrm{a}} L_{\mathrm{e}} L_{\mathrm{u}}=\frac{8 \pi G m\left(T^{3}\right)}{3\left[H\left(T^{3}\right)\right]^{2}},
$$

as for (2). This equation was ignored in Sect. 2.3, since it provides no constraint on $\Omega$. The Friedmann equation in this case is (4), which provides no constraint on the global parameters $L_{\mathrm{a}}, L_{\mathrm{e}}, L_{\mathrm{u}}$. Hence, two of these parameters are free. In general, the angles between the faces of the fundamental domain constitute additional free geometrical parameters, but for $L_{\mathrm{a}}, L_{\mathrm{e}}, L_{\mathrm{u}}$ defined orthogonally, these angles do not affect the volume of the manifold.

The parameter freedom for compact, flat FLRW models has frequently been thought of as an empirically undesirable property, since it makes the models easier to fit to observations, reducing the models' falsifiability. This freedom is not total. In the presence of an inhomogeneity, the residual gravity effect (Roukema et al., 2007; Roukema and Różański, 2009), can be invoked as a motivation for $L_{\mathrm{a}} \approx L_{\mathrm{e}} \approx L_{\mathrm{u}}$.

\subsection{The set $F_{m, H}^{2 r_{\mathrm{inj}}}$ of FLRW models parametrised by $2 r_{\mathrm{inj}}$}

However, without invoking the residual gravity effect, the parametrisation of compact, flat FLRW models can be made in a way that also applies to compact, curved FLRW models, by using a size parameter. Here, we propose twice the injectivity radius, i.e., the length of the shortest closed comoving spatial geodesic, written $2 r_{\text {inj }} R_{\mathrm{C}}$, where $2 r_{\text {inj }}$ is dimensionless, in the curved case, and $2 r_{\text {inj }}^{\prime}$, including a length dimension, in the flat case. This underestimates the full parameter freedom of the flat case, providing a conservative approach to constructing a measure space.

Equation (15) implies an upper limit to $2 r_{\mathrm{inj}}^{\prime}$. This occurs for the regular $\mathrm{T}^{3}$ model, in which $L_{\mathrm{a}}=L_{\mathrm{e}}=L_{\mathrm{u}}$, so that

$$
2 r_{\text {inj }}^{\prime}=\min \left(L_{\mathrm{a}}, L_{\mathrm{e}}, L_{\mathrm{u}}\right)=\left\{\frac{8 \pi G m\left(\mathrm{~T}^{3}\right)}{3\left[H\left(\mathrm{~T}^{3}\right)\right]^{2}}\right\}^{1 / 3} .
$$

Increasing any of the dimensions decreases at least one of the other dimensions, forcing $2 r_{\text {inj }}^{\prime}$ to decrease. A reasonable empirical lower bound for $2 r_{\text {inj }}^{\prime}$ can also be set, e.g. a comoving scale of $\sim 10 h^{-1}$ Gpc. Aurich (2008) estimate $2 r_{\text {inj }}=11.5 \pm$ $0.3 h^{-1} \mathrm{Gpc}$ from applying the cross-correlation Monte Carlo Markov chain method 
Roukema. Buliński. Szaniewska and Gaudin, 2008; Roukema. Buliński and Gaudin, 2008) to the 5-year WMAP data for a $T^{3}$ model. Hence, for the $T^{3}$ and related compact models with rectangular-prism fundamental domains

$$
\begin{aligned}
10 a\left(t_{\mathrm{t}}\right) h^{-1} \mathrm{Gpc} & \lesssim 2 r_{\mathrm{inj}}^{\prime} \\
& =\min \left(L_{\mathrm{a}}, L_{\mathrm{e}}, L_{\mathrm{u}}\right) \\
& \leq\left(\frac{8 \pi G}{3}\right)^{1 / 3} \max _{i=1,2,3}\left\{\frac{m\left(\mathrm{E}_{i}\right)}{\left[H\left(\mathrm{E}_{i}\right)\right]^{2}}\right\}^{1 / 3},
\end{aligned}
$$

where $\mathrm{E}_{1}, \mathrm{E}_{2}, \mathrm{E}_{3}$ follow Table I of Riazuelo et al. (2004). The compact flat models with hexagonal-prism fundamental domains, $\mathrm{E}_{4}$ and $\mathrm{E}_{5}$, have the corresponding limit

$$
\begin{aligned}
10 a\left(t_{\mathrm{t}}\right) h^{-1} \mathrm{Gpc} & \lesssim 2 r_{\mathrm{inj}}^{\prime} \\
& \leq 2^{1 / 3} 3^{-1 / 6}\left(\frac{8 \pi G}{3}\right)^{1 / 3} \max _{i=4,5}\left\{\frac{m\left(\mathrm{E}_{i}\right)}{\left[H\left(\mathrm{E}_{i}\right)\right]^{2}}\right\}^{1 / 3}
\end{aligned}
$$

(Appendix A.1). The other compact, orientable, flat model, i.e. the Hantzsche-Wendt space, $\mathrm{E}_{6}$, has a smaller injectivity radius, maximised in the regular case, giving

$$
\begin{aligned}
10 a\left(t_{\mathrm{t}}\right) h^{-1} \mathrm{Gpc} & \lesssim 2 r_{\mathrm{inj}}^{\prime} \\
& \leq 2^{-1 / 3}\left(\frac{8 \pi G}{3}\right)^{1 / 3}\left\{\frac{m\left(\mathrm{E}_{6}\right)}{\left[H\left(\mathrm{E}_{6}\right)\right]^{2}}\right\}^{1 / 3}
\end{aligned}
$$

(Appendix A.2).

Hence, over all curvatures, we can classify the full set of compact FLRW models by $2 r_{\text {inj }}^{\prime}$ for the flat case and $2 r_{\text {inj }} R_{\mathrm{C}}$ for the curved cases. $F_{m, H}^{2 r_{\text {inj }}}$ can now be written

$$
\begin{aligned}
F_{m, H}^{2 r_{\text {inj }}}= & \left\{2 r_{\text {inj }}^{\prime}: 10 a\left(t_{\mathrm{t}}\right) h^{-1} \mathrm{Gpc}<2 r_{\mathrm{inj}}^{\prime} \leq\left(\frac{8 \pi G}{3}\right)^{1 / 3} \eta\right\} \\
& \bigcup\left\{2 r_{\mathrm{inj}}(M) R_{\mathrm{C}}(M): M \in F, \widetilde{M} \in\left\{\mathbb{H}^{3}, S^{3}\right\}\right\},
\end{aligned}
$$

where

$$
\begin{aligned}
\eta:= & \max \left(\left\{\frac{m\left(\mathrm{E}_{i}\right)}{\left[H\left(\mathrm{E}_{i}\right)\right]^{2}}\right\}^{1 / 3}, i=1,2,3\right\} \\
& \bigcup\left\{2^{1 / 3} 3^{-1 / 6}\left\{\frac{m\left(\mathrm{E}_{i}\right)}{\left[H\left(\mathrm{E}_{i}\right)\right]^{2}}\right\}^{1 / 3}, i=4,5\right\} \\
& \left.\bigcup\left\{2^{-1 / 3}\left\{\frac{m\left(\mathrm{E}_{6}\right)}{\left[H\left(\mathrm{E}_{6}\right)\right]^{2}}\right\}^{1 / 3}\right\}\right) .
\end{aligned}
$$

As in the case of $F_{m, H}^{\Omega}(9)$, a single value of $2 r_{\text {inj }}^{\prime}$ or $2 r_{\text {inj }} R_{\mathrm{C}}$ can correspond to more than one exact-FLRW model.

\subsection{Measure spaces and probability spaces}

Let us set $\Sigma$ to be the smallest $\sigma$-algebra that contains all open sets on $F_{m, H}^{\Omega}$ induced by the usual topology on $\mathbb{R}$. Then $\left(F_{m, H}^{2 r_{\text {inj }}}, \Sigma, \lambda\right)$, where $\lambda$ is the Lebesgue measure, is 
a measure space over $2 r_{\text {inj }}^{\prime}$, twice the injectivity radius of exact-FLRW models of the Universe. Let us normalise this by defining

$$
\widehat{\lambda}=\frac{\lambda}{\left(\frac{8 \pi G}{3}\right)^{1 / 3} \eta-10 a\left(t_{\mathrm{t}}\right) h^{-1} \mathrm{Gpc}},
$$

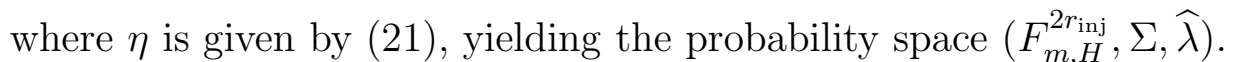

Hence, under the obvious probability space over the size of the 3-spatial section of FLRW models, the probability of a flat FLRW model is unity and the probability of a curved FLRW model is zero:

$$
\begin{aligned}
& \widehat{\lambda}\left(\left.2 r_{\text {inj }}\right|_{\Omega=1}\right)=1, \\
& \widehat{\lambda}\left(\left.2 r_{\text {inj }}\right|_{\Omega \neq 1}\right)=0 .
\end{aligned}
$$

This follows from (201) and (22), since the Lebesgue measure on an interval on the real line is the length of that interval, and the Lebesgue measure on a set of discrete, isolated points is zero.

An event that occurs with a probability of one is referred to as being almost sure (a.s.) in the Kolmogorov construction of probability spaces. This should not be confused with certainty. For example, an event of a uniform random process that selects a value from the interval $0 \leq x \leq 1$ on the real line results in a specific value $x^{*}$, despite the fact that the process a.s.t does not choose that specific value $x^{*}$. Thus, an a.s. outcome is not certain, even though its probability is one. In the case of interest here, if the Lebesgue measure is adopted and normalised, then a flat model a.s. occurs and a non-flat model a.s. does not occur.

\subsection{How can $\Omega=1$ occur almost surely?}

The probability space over $\Omega$, i.e. $\left(F_{m, H}^{\Omega}, 2^{F_{m, H}^{\Omega}}, \widehat{\mu}\right)$, follows from the link between local and global definitions of the density parameter, i.e. between curvature and topology, implied by Hypothesis 1, This relation can be described in terms of the curvature radius $R_{\mathrm{C}}$, as shown above. Thus, equality of the local and global definitions of $\Omega$ discretises it. In the flat case, the curvature is zero and $R_{\mathrm{C}}$ is undefined. The mathematical nature of flat, constant curvature 3 -manifolds allows them a continuous range of fundamental domain shapes. The physical processes that are presumed to select a 3-manifold from among those mathematically available are not constrained to choose fundamental domain size parameters from a discrete set. Unless a physical constraint is added, a continuous, finite range in $2 r_{\text {inj }}^{\prime}$, the size parameter adopted here, is allowed.

On the other hand, constant-curvature, curved 3-manifolds do not allow $2 r_{\text {inj }}$ to vary. The same rigidity that suggests the discrete measure in $\left(F_{m, H}^{\Omega}, 2^{F_{m, H}^{\Omega}, \widehat{\mu}}\right)$ leads even further, to the Lebesgue measure in $\left(F_{m, H}^{2 r_{\text {inj }}}, \Sigma, \widehat{\lambda}\right)$. Another way of saying this is that Hypothesis 1 not only links together the curvature and topology of exact-FLRW models, so that the rigidity of curved 3-manifolds constrains them to a discrete parameter space, $\dagger \dagger$ Almost sure and almost surely are both abbreviated as a.s. 
but it also frees up flat 3-manifolds to occupy a continuous parameter space, if the parameter chosen is, for example, the injectivity radius. This is why, given Hypothesis 1, the natural choice of measure leads to a probability space where a flat model a.s. occurs $(P \equiv \widehat{\lambda}=1)$ and a non-flat model a.s. does not occur $(P \equiv \widehat{\lambda}=0)$.

\section{Conclusions}

Cosmic topology has been referred to in the cosmological context in the pre-relativistic era (Schwarzschild, 1900) and by de Sitter (1917), Friedmann (1923, 1924), Lemaître (1931) and Robertson (1935) during the founding of relativistic cosmology. When the topology of the FLRW models is taken into account, the popular idea that $\Omega=1$ FLRW models constitute a class of measure zero among the full set of exact-FLRW models is no longer self-evident. This has been shown here by proposing that the parameters determined at a given epoch $t_{\mathrm{t}}$ following topology evolution, i.e. as the results of the processes of primordial spatial 3-manifold evolution (quantum or otherwise), are the global (by volume), total (by component) mass-energy $\left.m(M)\right|_{t_{\mathrm{t}}}$ and the Hubble parameter $\left.H(M)\right|_{t_{\mathrm{t}}}$, i.e. these are functions of the 3-manifold $M$ selected by those processes, while the density $\Omega$ is relegated to a derived parameter (Hypothesis 1).

This hypothesis leads to quite different measure spaces and associated probability spaces than those in which $\Omega=1$ FLRW models constitute a class of measure zero. When $\Omega$ is used to parametrise the FLRW models, the hypothesis leads to the discrete measure as the obvious choice. In this case, there is no obvious constraint requiring $\mu(\Omega=1)=0$. Moreover, use of the injectivity radius $r_{\text {inj }}$ to parametrise FLRW models, rather than $\Omega$, suggests the Lebesgue measure as the natural measure. In this case, the measure of the class of non-flat models is zero. Since the measure is normalisable, the probability of a flat FLRW model in the corresponding probability space is unity. Hence, in this case, a flat model occurs a.s. and a non-flat model a.s. does not occur, in the Kolmogorov probability sense of these terms. This is the reverse of what has been thought to be the case when topology is ignored.

How does this happen? For exact-FLRW models with compact spatial sections, the density parameter has both a local and a global physical meaning. The requirement of equality between the two definitions of the density parameter, and the rigidity of curved, constant-curvature 3-manifolds, reduce the parameter freedom of the non-flat models. In contrast, the lack of rigidity of the flat models allows them a continuum of possible sizes. Hence, the flat models become much more probable. This approach suggests a motivation independent of inflationary scenarios for the recent work finding that a $\mathrm{T}^{3}$ FLRW model provides a good fit to the WMAP sky maps (Spergel et al., 2003; Aurich et al., 2007; Aurich, 2008; Aurich et al., 2008, 2010). Moreover, a physical relation between the curvature and topology of comoving space is implied. 


\section{Acknowledgments}

Thank you to Karolina Zawada, Bartosz Lew and anonymous referees for several useful comments. Some of this work was carried out within the framework of the European Associated Laboratory "Astrophysics Poland-France". Use was made of the computer algebra program MAXIMA, the GNU OCTAVE command-line, high-level numerical computation software (http://www.gnu.org/software/octave), and the Centre de Données astronomiques de Strasbourg (http://cdsads.u-strasbg.fr).

\section{References}

Anderson M, Carlip S, Ratcliffe J G, Surya S and Tschantz S T 2004 CQG 21, 729, [arXiv:gr-qc/0310002].

Aurich R $2008 C Q G$ 25, 225017, [arXiv:0803.2130].

Aurich R, Janzer H S, Lustig S and Steiner F 2008 Classical and Quantum Gravity 25(12), 125006, [arXiv:0708.1420].

Aurich R, Lustig S and Steiner F 2005a CQG 22, 3443, [arXiv:astro-ph/0504656].

Aurich R, Lustig S and Steiner F $2005 b C Q G$ 22, 2061, [arXiv:astro-ph/0412569].

Aurich R, Lustig S and Steiner F 2010 CQG 27, 095009, [arXiv:0903.3133].

Aurich R, Lustig S, Steiner F and Then H 2007 CQG 24, 1879-1894, [arXiv:astro-ph/0612308]

Blanlœil V and Roukema B F, eds 2000 Paris: Blanlœil \& Roukema, [arXiv:astro-ph/0010170].

Caillerie S, Lachièze-Rey M, Luminet J P, Lehoucq R, Riazuelo A and Weeks J 2007 $A \mathscr{S} A$ 476, 691, [arXiv:0705.0217v2].

Cornish N J, Spergel D N, Starkman G D and Komatsu E 2004 Phys. Rev. Lett. 92, 201302, [arXiv:astro-ph/0310233].

Coule D H 1995 Classical and Quantum Gravity 12, 455-469, [arXiv:gr-qc/9408026]. de Sitter W 1917 MNRAS 78, 3-28.

Dowker F and Surya S 1998 Phys. Rev. D 58, 124019, [arXiv:gr-qc/9711070].

Ellis G F R 2006 ArXiv Astrophysics e-prints, [arXiv:astro-ph/0602280].

Ellis G F R and van Elst H 1999 in M Lachièze-Rey, ed., 'NATO ASIC Proc. 541: Theoretical and Observational Cosmology' pp. 1-116, [arXiv:gr-qc/9812046].

Euler L 1736 Commentarii Academiae Scientiarum Imperialis Petropolitanae 8, 128. http://www.math.dartmouth.edu/ euler/docs/originals/E053.pdf

Evrard G and Coles P 1995 Classical and Quantum Gravity 12, L93-L97, [arXiv:astro-ph/9507020].

Friedmann A 1923 Mir kak prostranstvo i vremya (The Universe as Space and Time) Leningrad: Academia. 
Friedmann A 1924 Zeitschr. für Phys. 21, 326.

Gabai D, Meyerhoff R and Milley P 2009 J. Amer. Math. Soc. 22, 1157, [arXiv:0705.4325].

Gausmann E, Lehoucq R, Luminet J P, Uzan J P and Weeks J 2001 CQG 18, 5155, [arXiv:gr-qc/0106033].

Gibbons G W, Hawking S W and Stewart J M 1987 Nuclear Physics B 281, 736-751.

Gibbons G W and Turok N 2008 Phys. Rev. D 77(6), 063516, [arXiv:hep-th/0609095].

Gundermann J 2005 ArXiv e-prints, [arXiv:astro-ph/0503014].

Hawking S W 1984 Nuclear Physics B 244, 135.

Key J S, Cornish N J, Spergel D N and Starkman G D 2007 Phys. Rev. D 75(8), 084034, [arXiv:astro-ph/0604616].

Kirchner U and Ellis G F R 2003 Classical and Quantum Gravity 20, 1199-1213.

Lachièze-Rey M and Luminet J 1995 Phys. Rep. 254, 135, [arXiv:gr-qc/9605010].

Lehoucq R, Lachièze-Rey $\mathrm{M}$ and Luminet $\mathrm{J} \quad \mathrm{P} \quad 1996$ A $6 A$ 313, 339, [arXiv:gr-qc/9604050].

Lemaître G 1931 MNRAS 91, 483-490.

Lew B and Roukema B F 2008 A $6 A$ 482, 747, [arXiv:0801.1358].

Luminet J P 1998 Acta Cosmologica XXIV-1, 105, [arXiv:gr-qc/9804006].

Luminet J and Roukema B F 1999 in M Lachièze-Rey, ed., 'NATO ASIC Proc. 541: Theoretical and Observational Cosmology. Publisher: Dordrecht: Kluwer,' p. 117, [arXiv:astro-ph/9901364].

Luminet J, Weeks J R, Riazuelo A, Lehoucq R and Uzan J 2003 Nature 425, 593, [arXiv:astro-ph/0310253].

Matveev S V and Fomenko A T 1988 Russian Math. Surveys 43, 3.

Mostow G D 1968 Publ.math.IHES 34, 53.

http://www.numdam.org/item?id=PMIHES_1968__34__53_0

Niarchou A and Jaffe A 2007 Phys. Rev. Lett. 99(8), 081302, [arXiv:astro-ph/0702436].

Rebouças M J and Gomero G I 2004 Braz. J Phys. 34, 1358, [arXiv:astro-ph/0402324].

Riazuelo A, Weeks J, Uzan J, Lehoucq R and Luminet J 2004 Phys. Rev. D 69, 103518, [arXiv:astro-ph/0311314].

Robertson H P 1935 ApJ 82, 284.

Roukema B F 2000 Bull. Astr. Soc. India 28, 483, [arXiv:astro-ph/0010185].

Roukema B F, Bajtlik S, Biesiada M, Szaniewska A and Jurkiewicz H 2007 A\&A 463, 861-871, [arXiv:astro-ph/0602159].

Roukema B F, Buliński Z and Gaudin N E 2008 A $\& A$ 492, 673, [arXiv:0807.4260]. 


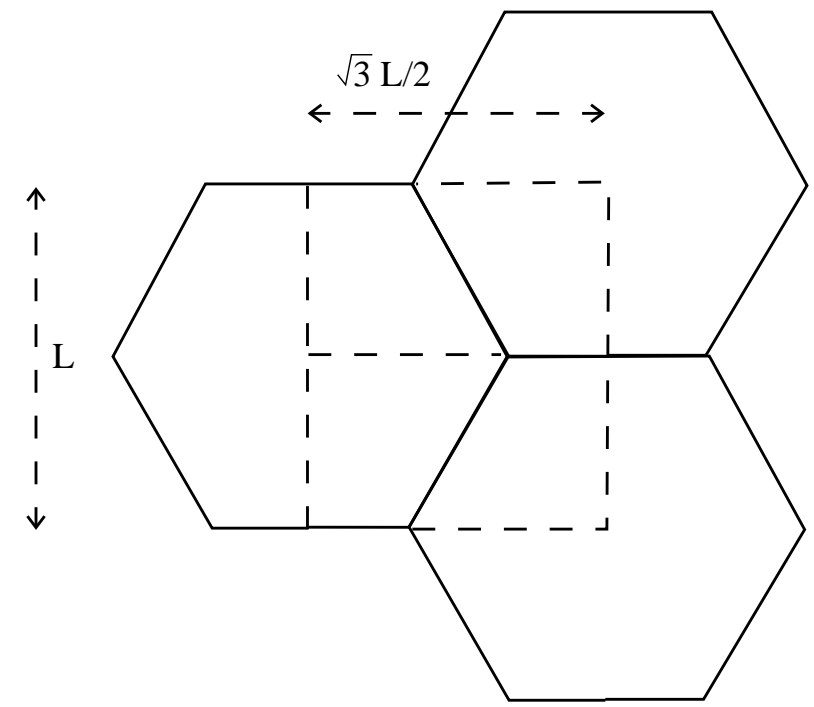

Figure 3. Orthogonal projection in $z$ direction of one copy of the fundamental domain (FD) of a hexagonal-prism (third-turn or sixth-turn) space illustrating its volume, given that the injectivity radius (horizontal and vertical shortest closed geodesic) is $L$. The hexagonal area can be cut and paste into an $L \times \sqrt{3} L / 2$ rectangle.

Roukema B F, Buliński Z, Szaniewska A and Gaudin N E 2008 A $ध A$ 486, 55, [arXiv:0801.0006].

Roukema B F and Różański P T 2009 A\&A 502, 27, [arXiv:0902.3402].

Schwarzschild K 1900 Vier.d.Astr.Gess 35, 337.

Seriu M 1996 Phys. Rev. D 53, 6902, [arXiv:gr-qc/9603002v1].

Spergel D N, Verde L, Peiris H V, Komatsu E, Nolta M R, Bennett C L, Halpern M, Hinshaw G, Jarosik N, Kogut A, Limon M, Meyer S S, Page L, Tucker G S, Weiland J L, Wollack E and Wright E L 2003 ApJSupp 148, 175, [arXiv: astro-ph/0302209]. Starkman G D 1998 CQG 15, 2529-2538.

Stewart J M, Stewart M E and Schwarzschild K 1998 CQG 15, 2539.

Vardanyan M, Trotta R and Silk J 2009 MNRAS 397, 431-444, [arXiv:0901.3354].

Weeks J 1985 PhD thesis Princeton University, (1985).

Weeks J, Luminet J P, Riazuelo A and Lehoucq R 2004 MNRAS 352, 258, [arXiv:astro-ph/0312312].

\section{Appendix A. Injectivity radii}

Appendix A.1. Injectivity radius of hexagonal-prism spaces

Figure 3 shows a projection of the fundamental domain (FD) of a hexagonal-prism space, with dimensions that maximise the injectivity radius for a fixed volume, i.e. the 


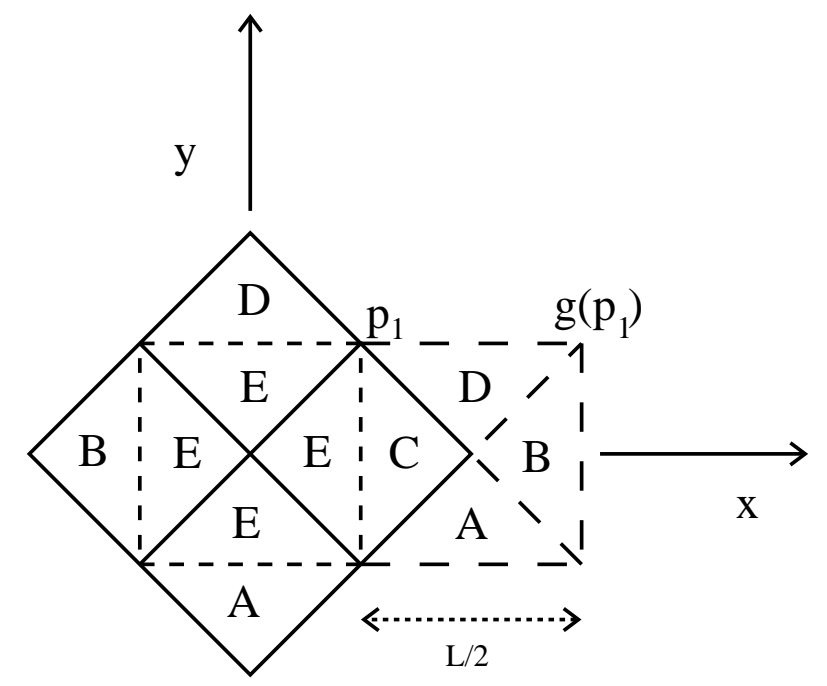

Figure 4. Orthogonal projection in $z$ direction of one copy of the fundamental domain (FD) of the Hantzsche-Wendt space illustrating its volume and injectivity radius. Looking down on the $\mathrm{FD}$ from high $z$, four rhombic (diamond) faces, AE, $\mathrm{BE}, \mathrm{DE}$, and $\mathrm{CE}$, are visible as projections to squares (solid lines). The FD can be thought of as a cube of side length $L / 2$ inscribed in the FD, surrounded by lateral square-pyramidal extensions A, B, C, and D, a superior square-pyramidal extension $\mathrm{E}$, and a corresponding one below the cube (not shown). Positions to which some of these can be pasted for volume calculation are shown by long-dashed lines. The $X$ and $Y$ axes' zero points are at the centre of the FD. Point $p_{1}$ is mapped to $g\left(p_{1}\right)$ by the holonomy $g$ (A.3).

"vertical" and "horizontal" shortest closed geodesics are both of length $L$. The volume of the FD is $V=\sqrt{3} L^{3} / 2$, so

$$
\frac{2 r_{\text {inj }}}{V^{1 / 3}}=2^{1 / 3} 3^{-1 / 6}
$$

Appendix A.2. Injectivity radius of Hantzsche-Wendt space

Figure 4 shows a projection of the FD of a regular (side length $L / 2:=L_{\mathrm{a}} / 2=L_{\mathrm{e}} / 2=$ $\left.L_{\mathrm{u}} / 2\right)$ Hantzsche-Wendt space, $E_{6}$ in Table I of Riazuelo et al. (2004). Five of the square-pyramidal extensions around the inscribed cube, A, B, D, E, and the unshown lower square-pyramidal extension can be cut off and pasted around the sixth one C, making a second cube of identical size. Hence, the FD volume is

$$
V=2(L / 2)^{3} \text {. }
$$

Let us, w.l.o.g., choose holonomy $g$ to be the first mapping in (48) in Riazuelo et al. (2004)

$$
g:(x, y, z) \mapsto(x+L / 2,-y+L / 2,-z) .
$$

Since the shift in the $x$ direction is identical for all points in the FD, $2 r_{\text {inj }}$ cannot be smaller than $L / 2$. The point $p_{1}=(L / 4, L / 4,0)$ in Fig. 4 is mapped to $g\left(p_{1}\right)=$ 
$(3 L / 4, L / 4,0)$, i.e. by a distance of $L / 2$. Thus, the $L / 2$ lower bound is attained. Hence,

$$
\frac{2 r_{\text {inj }}}{V^{1 / 3}}=\frac{L / 2}{2^{1 / 3}(L / 2)}=2^{-1 / 3} .
$$

